\title{
Direct Mapping of Confined Interfacial States in Oxides Using Aberration Corrected STEM and EELS
}

\author{
A. B. Shah ${ }^{1,2}$, J. M. Zuo ${ }^{1,2}$, Q. M. Ramasse ${ }^{3}$, J. G. Wen ${ }^{2}$, X. Zhai ${ }^{2,4}$, A. Bhattacharya ${ }^{5}$ J. N. \\ Eckstein $^{2,4}$ \\ ${ }^{1}$ Dept. of Mats. Sci. and Engineering, University of Illinois at Urbana-Champaign, Urbana, IL 61801 \\ ${ }^{2}$ F. Seitz Materials Research Lab., University of Illinois at Urbana-Champaign, Urbana, IL 61801 \\ ${ }^{3}$ NCEM, Lawrence-Berkeley National Laboratory, Berkeley, CA 94720 USA \\ ${ }^{4}$ Department of Physics, University of Illinois at Urbana-Champaign, Urbana, IL61801 \\ ${ }^{5}$ Materials Science Division and Center for Nanoscale Materials, Argonne National Laboratory, \\ Argonne, IL 60439
}

Oxide interfaces have attracted much attention in recent years due to the discovery of enhanced properties from interfacial electronic reconstruction, such as enhanced conductivity, magnetic ordering, and colossal magnetoresistance $[1,2]$. There has been much effort in engineering the interface sharpness to control these properties, as well as characterizing the interface sharpness, atom intermixing, and electronic states. Scanning transmission electron microscopy (STEM) coupled with electron energy loss spectroscopy (EELS) is a powerful method to probe the local electronic structure at the interfaces. Key developments in aberration correction have allowed for better than 1 $\AA$ STEM imaging resolution and an increased probe current over previous instruments $[3,4,5]$. The large probe current has led to better signal/noise ratio in EELS and improved sensitivity.

Here, we demonstrate the application of aberration corrected STEM and EELS for mapping confined, or localized interfacial states. We have investigated several oxide interfaces in the form of superlattices, including $\left(\mathrm{LaMnO}_{3}\right)_{2 \mathrm{n}}-\left(\mathrm{SrMnO}_{3}\right)_{\mathrm{n}}(\mathrm{LMO}-\mathrm{SMO})$ and $\left(\mathrm{LaMnO}_{3}\right)_{\mathrm{n}}-\left(\mathrm{SrTiO}_{3}\right)_{\mathrm{n}}(\mathrm{LMO}-$ STO). LMO and SMO are both antiferromagnetic insulators in bulk form. However, when combined in a superlattice, the interfaces can become ferromagnetic and conducting for $\mathrm{n}>3$. We have investigated an $11.8 \times$ 4.4 LMO-SMO superlattice, which has an asymmetric magnetization profile when cooled. The magnetization is enhanced at the sharp LMO-SMO interface and suppressed at the rough SMO-LMO interface. Using atomic resolution STEM and EELS, we have investigated structural and electronic asymmetries at the interfaces, and their relation to magnetic ordering. In particular, we looked into the fine structure of the oxygen $\mathrm{K}$ edges. Using the difference spectrum method, we detected evidence of interfacial states and measured its spatial distribution, which has been suggested by theory but the extent of the interfacial states has not been measured before.

The $(\mathrm{LMO})_{\mathrm{n}}-(\mathrm{STO})_{\mathrm{n}}$ superlattices are interesting for optical applications. LMO in bulk form has one broad optical absorption peak below $3.2 \mathrm{eV}$ and STO has no optical absorption below 3.2 $\mathrm{eV}$. Superlattices of these two films had additional optical absorption between 1.8 and $3.0 \mathrm{eV}$, depending on the thickness of the films [7]. Using atomic scale EELS in the STEM, we investigated the electronic states in the $n=2$ superlattice (Figure 1), which is epitaxially strained to the underlying STO substrate. We found direct EELS evidences of interfacial electronic states for band alignment and interfacial electron transfer, or charge leakage, in the $n=2$ superlattice. The band alignment, in the form of a $0.22 \mathrm{eV}$ chemical shift of $\mathrm{O} \mathrm{K}$ and $\mathrm{Ti} \mathrm{L}_{2,3}$ edges, was observed in ultrathin STO. The electron transfer from LMO to STO is evidenced by a reduction in the intensity of O $\mathrm{K}$ edge prepeak in STO, which is attributed to unoccupied $\mathrm{O} 2 \mathrm{p}$ states from hybridization with the Ti 
$3 \mathrm{~d}_{2 \mathrm{~g}}$ states. Our results show that the oxidation state in the fine structure of the $\mathrm{O} \mathrm{K}$ edge can be mapped with $4 \AA$ resolution, or better.

Electron probe aberration correction was essential for the high spatial resolution mapping of interfacial electronic states. However, localization of inelastic scattering limits the spatial resolution of EELS mapping. The oxide superlattices have a modulated structure which provide a way to quantify the EELS spatial resolution. The $2 \times 2$ LMO-STO superlattice has a large number of interfaces and favorably strong EELS signals and is ideal for studying EELS resolution. We show that the Ti L, Mn L, and La M edges EELS spatial resolution measured by the FWHM of the EELS signal is $4.1 \AA$, $3.4 \AA$, and $6.8 \AA$, respectively, in a $200 \mathrm{kV}$ aberration corrected STEM. The resolution of the Ti and $\mathrm{Mn} \mathrm{L}$ edges matches well with Egerton's empirical model [8], but the La M edge resolution is lower, due to strong elastic scattering from the heavy La columns.

Acknowledgements: Research is supported by the DOE BES DE-AC02-06CH11357 Digital Synthesis FWP and DEFG02-91ER45439. Microscopy was carried out at the Frederick Seitz Materials Research Laboratory Central Facilities, University of Illinois, which is partially supported by the U.S. Department of Energy under grants DE-FG02-07ER46453 and DE-FG02-07ER46471, and the National Center for Electron Microscopy, Lawrence Berkeley Lab, which is supported by the U.S. Department of Energy under grant DE-AC02-05CH11231.

\section{References}

[1] A. Ohtomo et al., Nature 419 (2002) 378 - 380.

[2] A. Bhattacharya et al., Phys. Rev. Lett. 100 (2008) 257203.

[3] P. E. Batson et al., Nature 418 (2002) 617.

[4] M. Haider et al., Ultramicroscopy 81 (2000) 163.

[5] O. L. Krivanek et al., Ultramicroscopy 96 (2003) 229.

[6] A. B. Shah et al., Adv. Mats. 22 (2010).

[7] X. Zhai et al., Adv. Mats. 22 (2010).

[8] R. F. Egerton, Ultramicroscopy 107 (2010) 575-586.

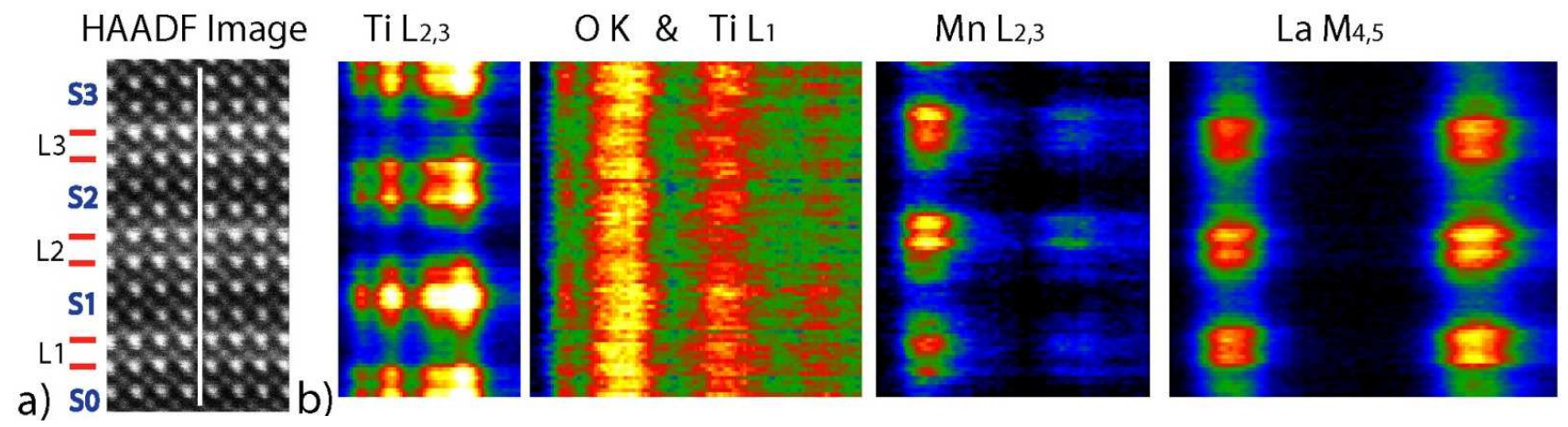

Figure 1: (a) Atomic resolution STEM image of a $\mathrm{SrTiO}_{3}-\mathrm{LaMnO}_{3}$ superlattice shows interface mixing of $\mathrm{La}$ and $\mathrm{Sr}$ in a single atomic layer. The darker films are $\mathrm{SrTiO}_{3}$ and brighter films $\mathrm{LaMnO}_{3}$. (b) Atomic scale EELS shows mapping of Ti, O, Mn and La with fine structural EELS. Notice there is Mn and Ti mixing between L1 and S0. These maps are used to measure the spatial resolution of EELS, which is lower in the $\mathrm{La} \mathrm{M}_{4,5}$ edge than Ti or $\mathrm{Mn} \mathrm{L}_{2,3}$ edges. 